Commentary/Jones: Human kinship, from conceptual stru

\section{Kin term diversity is the result of multilevel, historical processes}

\section{doi:10.1017/S0140525X10001962}

\section{Fiona M. Jordan and Michael Dunn}

Evolutionary Processes in Language and Culture, Max Planck Institute for Psycholinguistics, PB310, 6500AH Nijmegen, The Netherlands. fiona.jordan@mpi.nl michael.dunn@mpi.nl www.mpi.nl/people/jordan-fiona www.mpi.nl/people/dunn-michael

Abstract: Explanations in the domain of kinship can be sought on several different levels: Jones addresses online processing, as well as issues of origins and innateness. We argue that his framework can more usefully be applied at the levels of developmental and historical change, the latter especially. A phylogenetic approach to the diversity of kinship terminologies is most urgently required.

Kinship is unique as a domain of human experience for which we have a vast cultural and linguistic record. That record has languished unattended for too long, and Jones is to be commended for his attempt at reinvigorating kinship studies towards the understanding of our species-typical cognition. We have many points of agreement with the program laid out in the target article (and elsewhere: Jones 2000; 2003a; 2003b; 2004), especially with the general scientific aim of generating testable hypotheses from models and theory and seeing what the data have to say.

We agree with Jones that there are shortcomings in many approaches to kinship terminologies (sect. 2.3). None of these approaches provides a workable model that really captures what is going on in people's heads when they "do kinship" - or what goes on in speech communities when kinship terms change. Optimality Theory provides an ingenious approach to these problems, and the OT constraints that Jones proposes are for the most part plausibly motivated: They are based on universals of human experience, and they articulate human social priorities in the domain of kinship.

A weakness of Jones' account is that it is not clear about the timescale (or timescales) on which this kinship-directed version of OT operates. Within linguistics, OT is invoked at the timescale of online processing (McCarthy 2007b). Jones uses OT at this proximate level but also discusses OT as part of ultimate, "origin" explanations (cf. coordination games and the evolution of language, sect. 5.3). We question the plausibility of using OT at these two markedly different timescales and find it more satisfying to think of a generalised model operating in language acquisition and change. Kinship terminologies are semantic systems used by speech communities to coordinate social behaviours, and therefore in our view the relevant timescales at which the constraint rules of kinship operate are developmental - how do children acquire their culture-specific set of constraints and then employ them in the life course? - and historical - how do those culture-specific constraint arrangements change over time?

These two timescales are crucial components for good explanations of linguistic diversity. The variation in kinship terminology across human societies is not random (sect. 1) because, as Jones himself has outlined (Jones 2003a), it reflects Darwinian concerns - for example, sex, status, group membership, and so forth. There is a very small cross-cultural literature on the acquisition of kinship terminology, but developmentalists are unresolved on the relative importance of semantic complexity in kin terms versus the importance of the child's exposure to sets of relatives as referents (Benson \& Anglin 1987; Ragnarsdo'ttir 1997). In any case, to the extent that there are reliably recurring patterns of human infant experience, ontogenetic processes may further constrain the available variation. Most important, in any society the kinship terminology has a history: Individuals are not acquiring a terminology from, and languages are not constructing a system from, a space of infinite variation. This then re to grammar

immediately reduces the amount of variation that needs to be accounted for by the OT framework. Just as evolutionary developmental biologists have recognised the importance that both developmental constraints and historical evolutionary processes play in explaining organismal diversity (Breuker et al. 2006), so too are both important in understanding evolved diversity in kinship terminologies.

Evolutionary diversification produces hierarchically related taxa, and because these taxa cannot be considered independent data points, biologists have developed a range of computational phylogenetic methods that take history into account for comparative analyses. These methods have been successfully applied to linguistic and cultural evolution as well, notably with the construction of large-scale language phylogenies (e.g., Gray \& Atkinson 2003; Gray et al. 2009; Holden 2002; Kitchen et al. 2009). These trees provide statistical models of population history with which we can investigate cultural evolution: Hypotheses about coevolution, rates of change, directional models, ancestral states, borrowing, and the mode of evolution can all be addressed (e.g., Gray et al. 2007; Mace et al. 2005). Many studies have focused on kinship traits (Fortunato \& Mace 2009; Holden \& Mace 2003; Jordan et al. 2009), and we have begun to apply these methods to kinship terminologies in Austronesian and Bantu to test sequential models of sibling term evolution that are implied by markedness theory (Jordan, in press; forthcoming). We think that Jones' program holds the most promise if combined with a comparative phylogenetic approach that is implemented at the level of language change. One productive integration would allow us to detect if the outputs of constraint rerankings over time (i.e., rule changes throughout a language family) correlate with the empirical data when phylogeny is taken into account.

The constraints Jones proposes (Fig. 3 of the target article) are relatively uncontroversial as a starting point for describing the raw conceptual material, but historical affordances will determine the nature of how these play out in different language groups. Arguments about the primacy of these basic constraints therefore must wait until the empirical work is done. We need to understand the processes that have generated the observed cross-linguistic variation; these can inform speculation about what might be species-typical. As with other domains such as colour (Kay \& Regier 2003) or the human body (Majid et al. 2006) the nature of variation needs to be understood before we can make any grand or ultimate claims about universality and innateness in cognitive mechanisms. One of the attractions of the OT framework is that despite Jones' presentation it doesn't require our buy-in to any universal "atomic structures" of kinship (sect. 1). They may exist, but we don't need to appeal to them to explain the historically derived patterns of kinship terminologies across languages. 